\title{
On the Standardization of Semantic Web Services-based Network Monitoring Operations
}

\author{
Chengling Zhao ${ }^{1}$, Ziheng Liu ${ }^{2}$, Yanfeng Wang ${ }^{2}$ \\ The Department of Information Techonlogy, HuaZhong Normal University; \\ Wuhan,China,1zh20201@yahoo.com.cn,1zh20201@sohu.com, \\ yanfengwang123@sohu.com
}

Abstract. Web services have become an emerging XML-based approach in network management. And the combination of Web services and Semantic Web, in other words, Semantic Web services, make it possible to add semantic manners to services and automate network monitoring. This paper focuses on the standardization of network monitoring operations from the Semantic Web services' point of view. During this course, we especially take into account the problems about parameter transmission and operation granularity, and present the considerations about these problems in detail.

\section{Introduction}

Over many years, the Simple Network Management Protocol (SNMP) has become the most commonly used management protocol in IP network. However, these days, more focus is put on the XML-based approaches. And as one of the emerging XMLbased standards, Web services have been used in the network management. On the other hand, Semantic Web, known as the "Next Generation Internet", makes it possible to add semantic manners to services. Thus in this way, Semantic Web services can possibly automate network management.

Several studies have focused on the use of Web services in network management [1-3]. Additionally, a few researches have paid attention to the function of Semantic Web, especially Ontology, in network management [4,5].

However, few studies have turn to the potential of Semantic Web services in network monitoring. And our study just focuses on the use of Semantic Web services in the network monitoring operation instead of monitoring information. The goal of this paper is then to explain how standardization of monitoring operation can be 
achieved for Semantic Web services by the means of semantic markup languages for Web services, such as OWL-S in our study.

The organization of the paper is as follows. We will firstly present Semantic Web services background in Section 2, which is followed by Section 3 about the needs and the markup languages for standardization of network monitoring operations. With the OWL-S, we standardize Semantic Web services-based monitoring operations in Section 5, taking into account the parameter transmission and the operation granularity. We will conclude with Section 5 , which gives a summary of this paper.

\section{Background}

\subsection{Web services and SOA}

Web services is developed and standardized by the World Wide Web Consortium (W3C), which gives the following definition [6]: "A Web service is a software system designed to support interoperable machine-to-machine interaction over a network. It has an interface described in a machine-processable format (specifically WSDL). Other systems interact with the Web service in a manner prescribed by its description using SOAP-messages, typically conveyed using HTTP with an XML serialization in conjunction with other Web-related standards".

The word "services" in Web services refers to a Service-Oriented Architecture (SOA) [7]. In fact, SOA is a recent development in distributed computing, in which applications call functionality from other applications over a network. In an SOA, functionality is "published" on a network where two important capabilities are also provided - "discovery", the ability to find the functionality, and "binding", the ability to connect the functionality. So when considering a SOA, these three parts must be take into account, which are briefly presented as "publish", "find", and "bind".

In the Web services Architecture, three important roles are Web service provider, Web service requester, and Web service register, which correspond to the "publish", "find", and "bind" aspects of a SOA.

\subsection{Semantic Web}

As the "Next Generation Internet", the Semantic Web is a vision of the new architecture for the World Wide Web, characterized by the machine-accessible formal semantics added into traditional Web content.

As Tim Berners-Lee has presented, the Semantic Web is not a separate Web but an extension of the current one, in which information is given well-defined meaning, better enabling computers and people to work in cooperation [8]. The Semantic Web architecture put forward by Tim Berners-Lee in the academe is depicted in Fig. 1. 


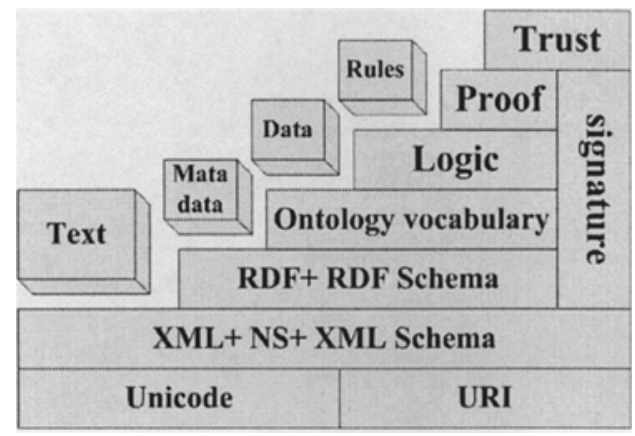

Fig 1. Semantic Web architecture

\subsection{Semantic Web services}

Semantic Web Services, as a new research paradigm, is generally defined as the augmentation of Web Service descriptions through Semantic Web annotations, to facilitate the higher automation of service discovery, composition, invocation, and monitoring in an open, unregulated, and often chaotic environment (that is, the Web) [9].

In fact, Semantic Web services represent an important step toward a new vision of the Semantic Web applied to Web services, in terms of utilizing, managing, and creating semantic markup languages for services.

Some experts have argued that, the relationship between the Semantic Web and the current Web Service architecture depends on your viewpoint [9]. In the near term, the deployment of Web Services may be critical, and Semantic Web techniques can enhance the current service architecture. In the long run, however, the Semantic Web vision itself may become more interesting, with Web Services offering an ubiquitous infrastructure on which to build the next generation of deployed multi-agent systems.

\section{Standardization for network monitoring}

\subsection{The needs}

In order to easily use Semantic Web services for network monitoring, there should be agreement on the monitoring information and operations. In other words, there is a need for standardization of information and operations.

Since standardization of information is a very broad subject and it is not directly related to Semantic Web services, we will abstract from it and just assume we have certain monitoring information defined in a MIB, such as in SNMP. The focus here will be on standardization of network monitoring operations. 


\subsection{The markup languages}

As for the standardization of network monitoring operations, an appropriate markup language is in great demand.

WSDL (Web Services Description Language) [10,11] only provides a mechanism to describe a Web service in a modular manner. This means that a WSDL document can be used jus in the case that the services are not automated.

But considering the semantic manners added into the Web services, we need a markup language particularly for Semantic Web services. As is shown in Fig. 2, we can see that XML, RDF and Ontology are three main layers for describing the semantics of Web information. However, RDF and RDF Schema, as well as XML and XML Schema, are not strong enough to express various resources and reason automatically. This has pushed the development of Ontology languages, from DAML (DARPA Agent Markup Language) [12] and OIL (Ontology Inference Layer) [13], to DAML + OIL (DARPA Agent Markup Language + Ontology Inference Layer) [14], and recently to OWL (Web Ontology Language) [14]. And OWL-S [16], an application of OWL, in particular, has evolved into a semantic markup language for Web services.

\section{Semantic Web Services-based network monitoring operations}

For standardization, we must consider two significant problems: parameter transmission and operation granularity.

\subsection{Parameter transmission}

Since OWL does not provide for the use of variables, there is no way to state in a class definition that one of the class properties is referenced elsewhere by a variable name, which makes the parameter transmission rather harder.

However, the use of parameter binding in a process definition will enable a specialized OWL-S process reasoner to use this information to determine which properties should have "the same value" in any coherent instance of the process being defined. In the notation of parameter binding, an instance of the class VALUEOF, with properties at Process and the Parameter denotes the object (value) of the specified parameter of the specified process.

On the other hand, parameters can also be combined and/or serialized in such a way, that the parameters are not described in the Service Model class. Merely their serialization is described in the class Input, a subclass of the class Parameter. Thus in this way, the input parameter is transmitted in data flow. This can be seen as lightweight parameter transparency.

An advantage of transparency is that monitoring information is abstracted from the process level, so the structure of information can change without having to modify the operation part. Since the transparent parameters are serialized in an XML structure, it can be contained in a class whose value is a string type. On both the manager and agent side, a generic XML parser can then be used to extract the parameters from the class. 


\subsection{Operation granularity}

In this study we have focused on the ifTable, which is part of the Interfaces Group MIB (IF-MIB) [17]. The ifTable contains data objects related to the state of all network interfaces available in the system on which the agent runs.

Using the ifTable, we have determined 4 distinct data retrieval schemes, each resulting in a different network monitoring agent. All granularities will be able to retrieve any data object from the ifTable; the distinction is that the structure and amount of data objects that are retrieved varies.

The first granularity is to retrieve all data objects separately. Our Semantic Web service that retrieves a single data object at a time is called GetIfCell.

The second granularity is to retrieve an entire row of data objects at a time, thus transmitting all available information on a single network interface. The Semantic Web service that retrieves a single row of data objects at a time is called GetIfRow.

The third granularity we have investigated is to retrieve an entire column of data objects at a time, thus transmitting the same piece of information for all network interfaces. The Web service that handles one column at a time will be called GetIfColumn.

The last granularity is to retrieve all data objects in the ifTable in a single operation. The Semantic Web service that retrieves all data objects in the ifTable at once is GetIfTable.

Then we can get a containment hierarchy of these four operations as a tree from relatively coarser operations to relatively finer operations. The containment tree is shown in Fig. 2.

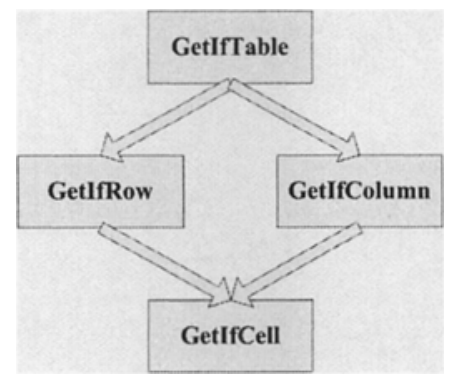

Fig 2. Containment tree of the four operations

\section{Conclusions}

In summary, standardizing Semantic Web services-based network monitoring operations can be done by the help of a semantic markup language for Web services, such as the OWL-S adopted in our study.

In the course of standardizing network monitoring operations, two important problems must be taken into account: parameter transmission and operation 
granularity. Parameter transmission can be achieved in OWL-S by the notation of parameter binding in the form of data flow, and the choice between non-transparent parameters and transparent parameters. As for the operation granularity, we take the IfTable for example and give four operations of different granularities, and argue that the choice for a certain operation granularity will be a tradeoff between simplicity and expressiveness.

The work presented here is part of ongoing research. We strongly encourage interested parties to react and comment on the discussed issues.

\section{References}

1. G. Pavlou, P. Flegkas, S. Gouveris, A. Liotta, On Management Technologies and the Potential of Web Services, IEEE Communication Magazine (July 2004), pp. 58-66.

2. J. Sloten, A. Pras, M. Sinderen, On the Standardisation of Web service management operations, Proc. 10th Open European Summer School (EUNICE 2004) and IFIP WG 6.3 Workshop(June 2004), pp. 143-150.

3. T. Drevers, R. Meent, A. Pras, Prototyping Web Services based Network Monitoring, Proc. 10th Open European Summer School (EUNICE 2004) and IFIP WG 6.3 Workshop(June 2004)pp. 135-142.

4. JE. López de Vergara, VA. Villagrá, JI. Asensio, B. Julio, Ontologies: Giving Semantics to Network Management Models, IEEE Network, special issue on Network Management, 17(3)(May/June 2003)pp. 15-21.

5. JE. López de Vergara, VA. Villagrá, B. Julio, Applying the Web Ontology Language to management information definitions, IEEE Communications Magazine, 42(7)(July 2004)pp. 68-74

6. W3C, Web services Architecture (8 August 2003), W3C Working Draft (August 2003):http:/www.w3.org/TR/2003/WD-ws-arch-20030808/\#whatis,

7. Service-Oriented Architecture (SOA), http:/www.service-architecture.com/.

8. T. Berners-Lee et al, The Semantic Web, Scientific American, 2001, 284(5), pp. 34-43..

9. T. Payne, O. Lassila, Semantic Web Services, IEEE Intelligent Systems, 19(4), (July/August 2004), pp. 14-15.

10. W3C, Web Services Description Language (WSDL) Version 2.0 Part 1: Core Language, W3C Working Draft (August 2005): http://www.w3.org/TR/2005/WD-wsd120-20050803,

11. Web Services Description Language (WSDL) Version 2.0 Part 2: Message Exchange Patterns, W3C Working Draft, (March 2004) :http ://www. w3.org/ TR/ 2004 /WD-wsdl20 patterns-20040326,

12. M.Dean DARPA AgentMarkup Language(DAML)

http://www.daml.org/2001/04/ssd-md/Overview.html.

13. OlL (Ontology Inference Layer), http:/www.ontoknowledge.org/oil/. 
14. T. Berners-Lee et al, DAML + OIL (DARPA Agent Markup Language + Ontology inference Layer), http://www.daml.org/2001/03/daml+oil-index.html.

15. W3C, OWL--Web Ontology Language Overview, W3C Recommendation, (February 2004)http:/www.w3.org/TR/2004/REC-owl-features-20040210/.

16. W3C, OWL-S: Semantic Markup for Services, W3C Member Submission(November 2004): http:/www.w3.org/Submission/2004/SUBM-OWL-S-20041122/,

17. K. McCloghrie, F. Kastenholz, The Interfaces Group MIB, IETF RFC 1902, June 2000. 